\title{
RATOS ESPONTANEAMENTE HIPERTENSOS E NEUROPATIAS PERIFÉRICAS
}

\author{
SPONTANEOUSLY HYPERTENSIVE RATS AND PERIPHERAL NEUROPATHIES
}

\author{
Valéria P S Fazan ${ }^{1}$, Andréa L R de Kalii², Adriana C L de Alcântara ${ }^{2}$, Adriana B Genari ${ }^{2}$, \\ Marcelo R Tavares ${ }^{2}$, Anaceres R Rodrigues ${ }^{2}$, Helio C Salgado ${ }^{3}$
}

\begin{abstract}
${ }^{1}$ Docente. Departamento de Cirurgia e Anatomia. ${ }^{2}$ Alunos de Pós-graduação. Departamento de Neurologia. ${ }^{3}$ Docente. Departamento de Fisiologia. Faculdade de Medicina de Ribeirão Preto - USP.

CoRResPondência: Valéria Paula Sassoli Fazan. Departamento de Cirurgia e Anatomia. Faculdade de Medicina de Ribeirão Preto - USP. Av. Bandeirantes 3900, Monte Alegre. CEP 14049-900 Ribeirão Preto - SP. Fone: (16) 3602-2501, FAX: (16) 3633-0017, e-mail: vpsfazan@yahoo.com.br ou vpsfazan@fmrp.usp.br
\end{abstract}

Fazan VPS, Kalil ALR, Alcântara ACL, Genari AB, Tavares MR, Rodrigues AR, Salgado HC. Ratos espontaneamente hipertensos e neuropatias periféricas. Medicina (Ribeirão Preto) 2006; 39 (1): 39-50.

Resumo: Modelo do Estudo: O presente estudo é uma revisão de literatura sobre o modelo de hipertensão espontânea e as conseqüências da hipertensão para o sistema nervoso periférico, somático e autonômico.

Importância do problema: Hipertensão é o principal fator de risco para acidente vascular cerebral e demência vascular, por causar importantes mudanças cerebrovasculares, tornando o cérebro propenso a infartos, microaneurismas e isquemias. As principais mudanças causadas no sistema nervoso central (SNC) pela hipertensão, incluem: diminuição do volume cerebral, aumento no volume dos ventrículos e perda neuronal. Além das alterações no cérebro, a hipertensão causa outros danos que culminam em uma série de alterações patológicas renais e outras doenças, as quais sustentam a elevação da pressão arterial, aumento da freqüência cardíaca, e aumento da resistência vascular periférica. O rato espontaneamente hipertenso (SHR) é reconhecido como um excelente modelo de hipertensão experimental e pode servir como modelo de estudos clínicos da hipertensão essencial humana. Embora esse modelo tenha sido bastante explorado em termos fisiológicos, estudos morfológicos, quando presentes, se limitam aos vasos. Mesmo quando nervos periféricos foram estudados morfologicamente nesses animais, os vasos epineurais, perineurais e endoneurais foram o alvo do estudo. Raros são os estudos que envolvem as fibras nervosas nesse modelo de hipertensão.

Comentários: Recentemente, estudamos as alterações do nervo depressor aórtico (NDA) em SHR. Nossos resultados mostraram redução do tamanho das fibras mielínicas e redução do tamanho e número das fibras amielínicas, comparados aos controles normotensos da linhagem Wistar-Kyoto. Outro estudo recente do nosso laboratório mostrou que, embora os níveis pressóricos dos SHR machos, bem como a freqüência cardíaca, sejam muito superiores aos das fêmeas, não há diferença morfológica nos nervos vagos cervicais entre SHR machos e fêmeas. Ainda, fazemos uma descrição morfológica e morfométrica do nervo sural de SHR, fornecendo parâmetros morfológicos para posteriores estudos funcionais.

Descritores: Hipertensão Experimental. Neuropatias Hipertensivas. Neuropatias Autonômicas. Nervo Depressor Aórtico. Nervo Sural. Nervo Vago. 


\section{1- RATOS ESPONTANEAMENTE HIPERTEN- SOS - SHR: MODELO DE HIPERTENSÃO}

Hipertensão é o principal fator de risco para acidente vascular cerebral e demência vascular, por causar importantes mudanças cerebrovasculares, tornando o cérebro propenso a infartos, microaneurismas e isquemias. As principais mudanças causadas no sistema nervoso central (SNC), pela hipertensão, incluem: diminuição do volume cerebral, aumento no volume dos ventrículos e perda neuronal ${ }^{1}$. Além das alterações no cérebro, a hipertensão causa outros danos que culminam em uma série de alterações patológicas renais e outras doenças ${ }^{2}$, as quais sustentam a elevação da pressão arterial, aumento da freqüência cardíaca, e aumento da resistência vascular periférica ${ }^{3}$.

Em 1963, Okamoto e Aoki ${ }^{4}$ introduziram um novo modelo experimental de hipertensão, sem que nenhum recurso fisiológico, farmacológico ou cirúrgico fosse necessário. Os ratos espontaneamente hipertensos - SHR foram desenvolvidos por uma reprodução genética meticulosa, entre irmãos e irmãs, que uniformemente resultou em animais naturalmente portadores de doença hipertensiva, em 100\% dos descendentes ${ }^{5}$. Desde então, o SHR é reconhecido como um excelente modelo de hipertensão experimental e pode servir como modelo de estudos clínicos da hipertensão essencial humana.

A semelhança entre a hipertensão humana e a observada no SHR, inclui: a) a predisposição genética para hipertensão sem etiologia específica, b) o aumento da resistência periférica total sem expansão de volume e, c) igual resposta a tratamentos com drogas ${ }^{6,7}$. Por apresentar um relativo curto período de vida, ser pequeno, ter relativamente baixo custo, e ser de fácil manutenção em biotérios, os SHR são utilizados para estudo da história natural, determinantes genéticos e alterações fisiopatológicas da doença hipertensiva essencial.

Autores ressaltam o cuidado na reprodução da linhagem de ratos hipertensos (SHR) e seus controles normotensos - ratos Wistar Kyoto (WKY), pois as características distintas de cada linhagem podem mudar levemente de uma geração para outra, ou entre diferentes colônias ${ }^{8,9}$. Todavia continuam acreditando na comparação entre mecanismos presso-reguladores da hipertensão essencial, e em vários modelos genéticos de hipertensão experimental.

Em relação ao desenvolvimento da hipertensão e das alterações da freqüência cardíaca em SHR jovens, Dickhout e Lee ${ }^{10}$, descreveram que a mensura- ção indireta da pressão arterial sistólica mostrou-se similar entre SHR e WKY na $2^{\mathrm{a}}$ e $3^{\mathrm{a}}$ semanas de vida. Pequena diferença, porém estatisticamente significante $(\mathrm{P}=0.02)$ apareceu na $4^{\mathrm{a}}$ semana $(\mathrm{SHR}=127 \pm 3 /$ $\mathrm{WKY}=118 \pm 3 \mathrm{mmHg}$ ) e, por volta da $6^{\mathrm{a}}$ semana, a pressão arterial sistólica em SHR foi significativamente elevada comparada a do WKY $(\mathrm{P}=0.005)$ $(\mathrm{SHR}=148 \pm 3 / \mathrm{WKY}=120 \pm 5 \mathrm{mmHg})$. A freqüência cardíaca foi significativamente maior em SHR comparada ao WKY nas $2^{\mathrm{a}}, 3^{\mathrm{a}}$ e $4^{\mathrm{a}}$ semanas $(\mathrm{SHR}=397 \pm 8 / 430 \pm 7 / 441 \pm 4$ e WKY $=373 \pm 9 /$ $380 \pm 5 / 407 \pm 5$, respectivamente), mas se tornou similar na $6^{\mathrm{a}}$ semana $(\mathrm{SHR}=398 \pm 5 / \mathrm{WKY}=392 \pm 7)$. Concluíram, que a freqüência cardíaca elevada precede a elevação da pressão arterial sistólica e uma taquicardia pré-hipertensiva provavelmente é o primeiro passo do desenvolvimento da hipertensão nos SHR.

Dois outros estudos ${ }^{2,7}$ descreveram que o período de maior aumento da pressão arterial sistólica em SHR é da $3^{\mathrm{a}}$ à $10^{\mathrm{a}}$ semana, e que esta pode aumentar até a $20^{\mathrm{a}}$ semana de idade. Esses autores descreveram que a média da pressão arterial sistólica para SHR de 20 a 24 semanas de idade é de aproximadamente 190 - 200 mmHg (mensuração direta), comparada com 115 - $130 \mathrm{mmHg}$ dos WKY.

Trippodo e Frohlich ${ }^{7}$ ressaltaram que, embora o SHR seja um excelente modelo da hipertensão essencial humana, há as seguintes ressalvas: 1) reconhecimento da improbabilidade de que ambas as formas de hipertensão espontânea (rato e o homem) sejam expressões idênticas de uma doença hipertensora determinada geneticamente; 2) ambas têm origem poligênica e são influenciadas por fatores ambientais; 3) sendo o controle cardiocirculatório multifatorial, certos mecanismos pressores não se expressam, necessariamente, em ambas as situações; 4) o conceito fisiológico de que o desequilíbrio de um mecanismo regulatório implica na expressão de um outro mecanismo, isto implica que alterações secundárias, por exemplo, adaptativas, devam ocorrer nos dois modelos. Independentemente da controvérsia quanto à similaridade, ou não, entre o SHR e a hipertensão essencial do homem, este modelo tem se revelado bastante útil para melhor compreensão de mecanismos fisiopatológicos, quer do próprio modelo em si, quer da hipertensão essencial do homem. Embora a hipertensão dos SHR seja de desenvolvimento espontâneo, fatores ambientais tais como, ingestão exagerada de sódio ${ }^{11}$, estresse ${ }^{12}$, alterações sociais ${ }^{13}$, e alterações do ciclo claro/escuro ${ }^{14}$ afetam esse desenvolvimento. 
A hipertensão do SHR adulto está associada com um aumento da resistência periférica total e um débito cardíaco normal ou diminuído ${ }^{15}$, embora nos animais mais jovens ( $<12$ semanas) tivessem sido observados débitos cardíacos normais ou aumenta$\operatorname{dos}^{16}$. Com o desenvolvimento da hipertensão arterial o SHR desenvolve uma progressiva hipertrofia cardíaca $^{17 / 20}$. O débito cardíaco permanece nos níveis normais com o progresso da hipertensão até que nos estágios finais a função cardíaca começa a ser comprometida, quando então, o débito cardíaco começa a reduzir em função de uma insuficiência cardíaca congestiva ${ }^{17}$. Com relação à freqüência cardíaca, esta se encontra mais elevada do que nos ratos WKY já na $3^{a}$ semana de vida e se correlaciona, positivamente, com os níveis de pressão atingidos até a $6^{\mathrm{a}}$ semana de vida $^{10}$. A taquicardia é considerada como resultado de um aumento na freqüência intrínseca do marca passo cardíaco. É interessante que a elevação precoce da freqüência cardíaca nos SHR é um índice altamente preditivo da ocorrência de hipertensão na população estudada ${ }^{10}$.

Conforme mencionado anteriormente um achado consistente nos SHR é o aumento da resistência periférica total ${ }^{21,22}$. Parece que as pequenas artérias, arteríolas e, possivelmente, os esfíncteres pré - capilares sejam os principais responsáveis pelo aumento da resistência vascular periférica ${ }^{7,23}$. Acredita-se que tanto processos ativos como estruturais, sejam responsáveis pelo aumento da resistência periférica. Por exemplo, alteração da geometria vascular ${ }^{18,23}$, alterações da membrana celular da musculatura lisa vascu$\operatorname{lar}^{24}$, ou diminuição da densidade arteriolar da musculatura esquelética ${ }^{25}$. Foi demonstrado, ainda, um aumento da resistência venosa ${ }^{26}$, possivelmente relacionada ao aumento da atividade simpática ${ }^{22,27} \mathrm{e} / \mathrm{ou}$ hipertrofia venular ${ }^{26}$. Estudos mais recentes ${ }^{28}$ mostraram que nos SHR as alterações das propriedades funcionais precedem o desenvolvimento da hipertensão arterial, e que a redução da distensibilidade e complacência vascular nos animais jovens resulta de uma hipertrofia da camada média ao invés de alterações intrínsecas das propriedades elásticas dos vasos.

A importância dos fatores neurais no desenvolvimento da hipertensão do SHR foi demonstrada por meio da redução da pressão arterial em resposta à ablação cirúrgica, ou farmacológica, do sistema nervoso autônomo simpático, prevenção do desenvolvimento da hipertensão por imunossimpatectomia, simpatectomia química e outros métodos ${ }^{7}$. Existe, por- tanto, uma ampla documentação de uma hiperatividade simpática no modelo SHR $22,27,29,30$. Entretanto, a similaridade das respostas $(\Delta \%)$ pressóricas e de resistência periférica ao hexametônio, entre os SHR e os normotensos WKY sugere algum outro mecanismo, além do simpático, participando na manutenção da hipertensão arterial de SHR jovens e velhos ${ }^{31}$.

\section{2- ESTUDOS MORFOLÓGICOS E FUNCIO- NAIS EM SHR}

Estudos do funcionamento do músculo cardíaco hipertrofiado, ou da regressão da hipertrofia com terapias anti-hipertensivas, são bem desenvolvidos nesse modelo experimental, face o desenvolvimento da hipertrofia ventricular ser uma resposta à lenta e progressiva elevação da pressão arterial, e da resistência periférica total ${ }^{32 / 35}$. Em relação ao músculo estriado, Kramer e Waldrop ${ }^{36}$ realizaram um estudo da resposta neuronal em SHR, durante contração muscular isométrica, e obtiveram o seguinte resultado: tanto os SHR quanto os WKY, apresentaram significante redução da pressão arterial, evocada pela contração muscular, via nervo tibial; porém, esta redução ocorreu muito rapidamente e não se manteve por toda a contração. Em WKY a média foi de $-21.1 \pm 1.8 \mathrm{mmHg}$, enquanto que nos SHR foi de $-33.2 \pm 1.9 \mathrm{mmHg}$. Não observaram alteração significativa nas freqüências cardíaca e respiratória nos grupos estudados.

Com relação a estudos morfológicos dos vasos, esses estão concentrados nas descrições comparativas das alterações vasculares entre SHR e WKY. Dickhout e Lee ${ }^{3}$ estudaram a estrutura e a função de pequenas artérias mesentéricas, em SHR de 3 e 4 semanas de vida, com pressão arterial similar. Esses autores concluíram que as alterações estruturais incluem: um aumento no volume da camada média e aumento no número de camadas de células musculares lisas, mas nenhuma mudança no diâmetro do lúmen no estado relaxado. Essas alterações estruturais tem manifestação funcional pelo fato que, as artérias estudadas nos SHR, sofreram aumento no número de contrações, resultando em um lúmen significativamente menor em resposta a estimulação por $\mathrm{KCl}$ ou norepinefrina. As artérias dos SHR pré-contraídas com $\mathrm{KCl}$, foram também capazes de manter um lúmen menor do que as artérias dos WKY, em pressões intramurais similares. Os SHR com 4 semanas de vida apresentaram uma resposta contrátil bem maior que os WKY. 
Um estudo mais recente, sobre as mudanças vasculares no nervo isquiático de SHR e WKY relatou que, o aumento da resistência dos vasos é a principal conseqüência de alterações estruturais nas artérias, relacionada à hipertensão. Essas alterações consistem no espessamento da parede da artéria e estreitamento do lúmen. Esse fenômeno, se não compensado, determina uma hipoperfusão e uma redução no suprimento de oxigênio para os tecidos. Os resultados demonstraram que as artérias interfasciculares e os vasos capilares intrafasciculares são sensivelmente diferentes entre si. Mediante a hipertensão, as artérias interfasciculares (epi e perineurais), reagem primeiramente, e ambos sofrem, em SHR, espessamento de parede e diminuição da contratilidade, pela redução do lúmen. Já os capilares intrafasciculares (endoneurais), reagem sofrendo mudanças na contratilidade (em vasos com diâmetros menores que $35 \mu \mathrm{m}$ ), porém não desenvolvem espessamento de parede ${ }^{37}$.

Persson et al..$^{38}$, estudaram a contribuição de mecanismos periféricos e espinais, relacionados com a hiperatividade simpática, apresentada pelos SHR. Eles descreveram que anormalidades vasculares são derivadas da hipertrofia e hiperplasia da célula muscular e hiper-inervação simpática. Uma quantidade elevada de norepinefrina e neuropeptídeo Y, foi encontrada em tecidos não vasculares de SHR, sugerindo que a hiper-inervação não está restrita ao tecido vascular, e pode incluir outros tecidos inervados simpaticamente, como a bexiga e a uretra. Concluíram, por exemplo que, as alterações na função da bexiga de SHR, parecem estar associadas com mudanças no controle noradrenérgico do reflexo de micção.

\section{3- NERVOS E HIPERTENSÃO}

A hipertensão arterial pode afetar a aferência, a eferência, bem como as conexões centrais do reflexo barorreceptor. Adaptações estruturais cardiocirculatórias devido ao enrijecimento dos vasos são consideradas como responsáveis pelas alterações das aferências barorreceptoras bem como das eferências do barorreflexo ${ }^{39}$. Entretanto, em hipertensos humanos e experimentais, o controle reflexo da freqüência cardíaca tem sua sensibilidade reduzida ${ }^{18,40}$ devido, principalmente, à atenuação da função vagal ao invés de alterações simpáticas ${ }^{18,41}$. Além disso, há evidências de que o desenvolvimento do déficit vagal nos SHR coincide com o início da hipertrofia cardíaca, e não com o início da hipertrofia vascular ${ }^{18}$. As aferências barorreceptoras do arco aórtico trafegam com o nervo depressor aórtico (nervo de Cyon, NDA) o qual se junta ao vago (X par craniano) para alcançar o sistema nervoso central. As eferências parassimpáticas trafegam no nervo vago.

Em $1999^{42}$, estudamos os aspectos morfológicos dos fascículos e das fibras mielínicas do NDA em SHR e em seus controles, ratos WKY (Figura 1). O estudo mostrou, por meio de morfometria computadorizada que, os NDA do lado esquerdo dos WKY, têm dimensões fasciculares transversais significativamente maiores, quando comparados aos dos SHR de mesma idade e que, as fibras mielínicas dos WKY, também apresentam diâmetros médios significativamente maiores que as dos SHR (Tabela I). Interessantemente, o número de fibras mielínicas não foi diferente entre os grupos, mas a densidade dessas fibras foi maior nos SHR. Esse resultado nos levou a concluir que o maior tamanho do fascículo dos NDA nos WKY estava relacionado ao aumento de algum outro componente do endoneuro, que não as fibras mielínicas. Também, o fato das fibras mielínicas dos SHR apresentarem menor diâmetro estaria contribuindo para essa característica das dimensões fasciculares dos NDA de SHR.

Posteriormente, descrevemos ${ }^{43}$ uma redução significativa no número de fibras amielínicas nos NDA de SHR, bem como do tamanho dessas fibras (Figura 1), comparados aos WKY (Tabela II). Esse achado explica a redução das dimensões fasciculares sem redução no número de fibras mielínicas, descrita anteriormente ${ }^{42}$. Discutimos que as diferenças observadas entre as duas linhagens podem contribuir, pelo menos em parte, para as alterações da função barorreceptora nos SHR. De fato, Takaeda et al..$^{44}$ estudaram a função dos ADN nos SHR por meio de deaferentação do $\mathrm{ADN}$, mostrando evidências de uma resposta simpatoinibitória reduzida à elevação da pressão arterial, por infusão de fenilefrina. Os autores sugeriram que um prejuízo da aferência barorreceptora poderia contribuir para o desenvolvimento da hipertensão nos SHR. Importante ressaltar que as fibras amielínicas apresentam papel importante na inibição tônica dos centros vasomotores bulbares, especialmente na presença de hipertensão ${ }^{45}$, o que poderia sugerir que o número reduzido de fibras amielínicas nos NDA de SHR poderia apresentar algum papel no desenvolvimento da hipertensão desses animais. Mais recentemente ${ }^{46}$, demonstramos que a relação entre a espessura da bainha de mielina e o diâmetro dos axônios mielinizados, está alterada nos NDA de SHR, quando 

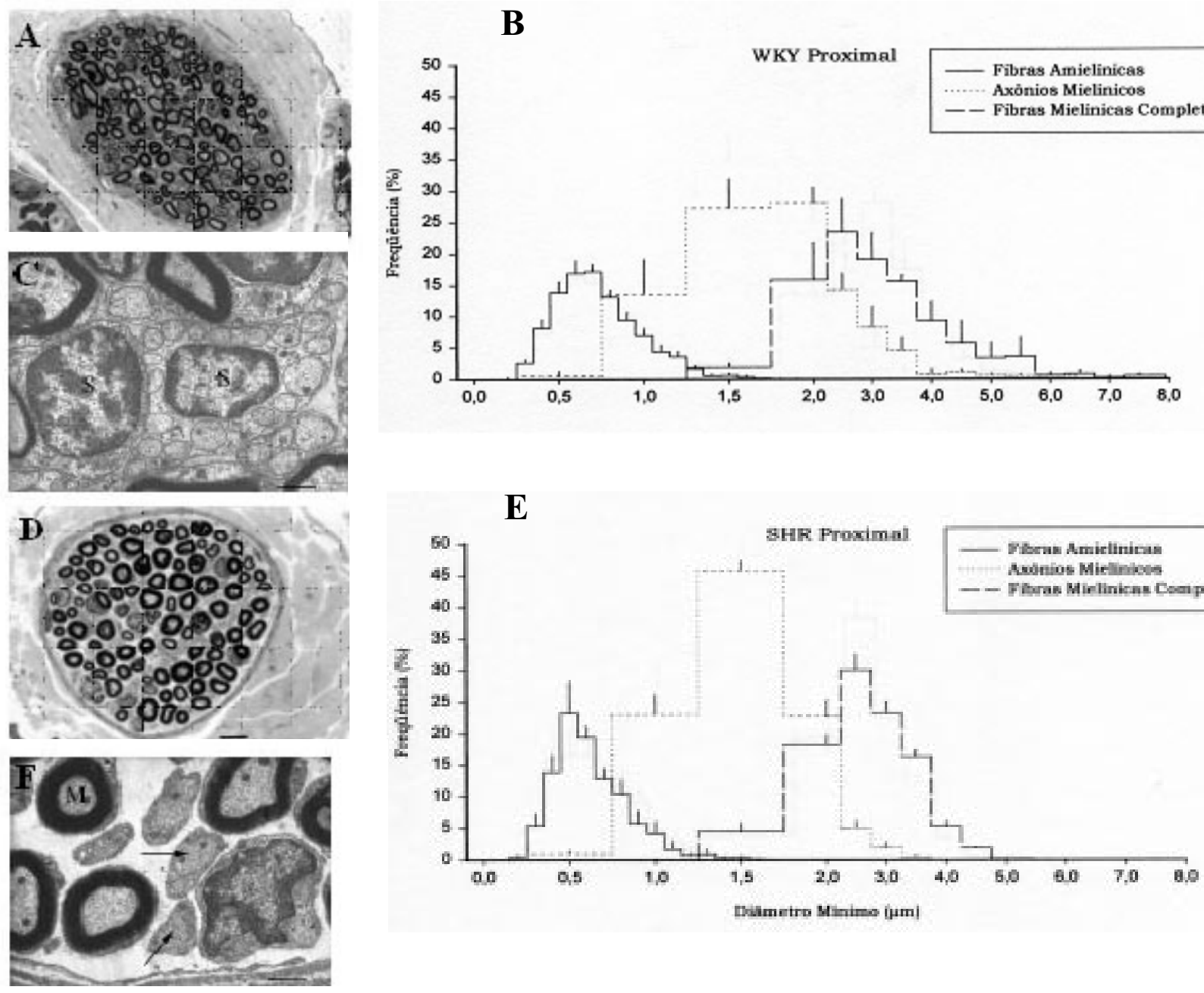

Figura 1: Secção transversal semi-fina (segmento proximal) de ADN de rato WKY (A), com a representação dos histogramas de distribuição dos diâmetros das fibras mielínicas (linha tracejada), dos respectivos axônios (linha pontilhada) e das fibras amielínicas (linha contínua) desses nervos (B), e uma secção transversal ultra-fina (microscopia eletrônica de transmissão) de uma área do endoneuro do respectivo nervo (C). Na comparação com os animais SHR, notar fibras mielínicas com bainha de mielina mais espessa, visível à microscopia de luz (D) e confirmada pela microscopia eletrônica de transmissão $(F)$. Notar também o desvio dos histogramas (E) para a esquerda nos SHR. Barra de escala em $A$ e $D=20 \mu \mathrm{m}$. Barra de escala em $C$ e $F=1 \mu \mathrm{m}$. $S=$ núcleo da célula de Schwann. $M=$ Fibra mielínica atrófica. Setas indicam fibras amielínicas, em número pequeno no endoneuro dos ADN de SHR, comparado aos WKY.

Tabela I: Parâmetros morfométricos dos fascículos dos NDA de SHR e WKY.

\begin{tabular}{|l|c|c|c|c|}
\hline & \multicolumn{2}{|c|}{ WKY (N=13) } & \multicolumn{2}{|c|}{ SHR (N=9) } \\
\hline & Proximal & Distal & Proximal & Distal \\
\hline Área Fascicular $\left(\mathrm{mm}^{2}\right)$ & $1,9 \pm 0,2$ & $1,9 \pm 0,2$ & $1,2 \pm 0,1^{*}$ & $1,4 \pm 0,3$ \\
\hline Diâmetro Fascicular $(\mu \mathrm{m})$ & $51 \pm 4$ & $47 \pm 3$ & $41 \pm 4^{*}$ & $43 \pm 7$ \\
\hline Número de fibras mielínicas & $83 \pm 10$ & $73 \pm 8^{* *}$ & $72 \pm 11$ & $74 \pm 9$ \\
\hline Número de fibras amielínicas & $335 \pm 68$ & $337 \pm 46$ & $130 \pm 14^{*}$ & $242 \pm 77^{*}$ \\
\hline Porcentagem de ocupação das fibras mielínicas (\%) & $42 \pm 5$ & $37 \pm 3$ & $37 \pm 3$ & $36 \pm 4$ \\
\hline Porcentagem de ocupação das fibras amielínicas (\%) & $12 \pm 3$ & $13 \pm 2$ & $5 \pm 1^{*}$ & $7 \pm 1 *$ \\
\hline N = número de animais estudados. * indica diferença entre as linhagens. ** indica diferença entre segmentos proximal e distal.
\end{tabular}


Tabela II: Parâmetros morfométricos das fibras mielínicas e amielínicas dos NDA de SHR e WKY.

\begin{tabular}{|l|c|c|c|c|}
\hline & \multicolumn{2}{|c|}{$\mathbf{W K Y}(\mathbf{N}=\mathbf{1 0})$} & \multicolumn{2}{c}{ SHR (N=9) } \\
\hline & Proximal & Distal & Proximal & Distal \\
\hline Diâmetro da fibra mielínica $(\mu \mathrm{m})$ & $3,0 \pm 0,1$ & $3,0 \pm 0,1$ & $2,5 \pm 0,04^{*}$ & $2,4 \pm 0,07^{*}$ \\
\hline Diâmetro do axônio mielinizado $(\mu \mathrm{m})$ & $1,7 \pm 0,1$ & $1,8 \pm 0,1$ & $1,3 \pm 0,04^{*}$ & $1,3 \pm 0,05^{*}$ \\
\hline Área da bainha de mielina $(\mu \mathrm{m} 2)$ & $5,9 \pm 0,4$ & $6,0 \pm 0,3$ & $4,6 \pm 0,5^{*}$ & $4,2 \pm 0,3^{*}$ \\
\hline Razão g & $0,6 \pm 0,01$ & $0,6 \pm 0,01$ & $0,5 \pm 0,01^{*}$ & $0,5 \pm 0,02^{*}$ \\
\hline Diâmetro da fibra amielínica $(\mu \mathrm{m})$ & $0,7 \pm 0,04$ & $0,7 \pm 0,03$ & $0,6 \pm 0,04^{*}$ & $0,6 \pm 0,04^{*}$ \\
\hline $\mathrm{N}$ = número de animais estudados. * indica diferença entre as linhagens. & & \\
\hline
\end{tabular}

comparados aos WKY. Uma relação linear entre a espessura da bainha de mielina e o diâmetro dos axônios mielinizados é esperada para nervos normais e, embora ainda linear, desvios da inclinação das retas de regressão podem indicar alterações axonais ou da bainha de mielina. No caso dos NDA de SHR, a alteração dessa relação foi sugestiva de atrofia axonal ${ }^{46}$. Geralmente, acredita-se que o diâmetro axonal seja o parâmetro morfométrico mais importante para a velocidade de condução ${ }^{47}$. O conjunto de informações morfológicas e morfométricas obtidas nos NDA dos SHR sugere que, esses nervos possam estar conduzindo informações para o SNC com velocidade menor que dos WKY, o que pode causar uma demora na chegada das informações barorreceptoras no núcleo do trato solitário (NTS).

Importante ressaltar que os estudos que mostraram diferenças morfológicas e morfométricas entre os NDA de SHR e WKY foram realizados em ratos machos e fêmeas, de mesma idade, porém sem distinção quanto ao gênero. Esse fato despertou a necessidade de uma investigação sobre eventuais diferenças morfológicas e morfométricas entre gêneros nos SHR, uma vez que existem diferenças reconhecidas nos níveis de pressão arterial entre machos e fêmeas de mesma idade. Dessa forma, uma investigação morfológica e morfométrica recente, foi conduzida em nosso laboratório, em nervos vagos de SHR machos e fêmeas, com hipertensão arterial bem estabelecida (20 semanas de vida). A primeira observação morfológica importante é de que o nervo vago de SHR não difere dos nervos vagos dos WKY e de outras linhagens de ratos (Figura 2). Nossos dados de- monstram que, embora os machos apresentem níveis pressóricos significativamente mais elevados que as fêmeas de mesma idade ( $200 \pm 7$ vs. $180 \pm 6 \mathrm{mmHg}$ ), os dados morfométricos fasciculares, bem como das fibras mielínicas, não são diferentes (Tabela III). Esses dados representam a primeira descrição morfométrica dos nervos vagos de SHR e fornecerão bases morfológicas para o melhor entendimento da função das eferências barorreceptoras parassimpáticas. Mais ainda, esses resultados definem que, após o estabelecimento da hipertensão arterial, não existem diferenças morfológicas nos nervos vagos, por onde trafegam as eferências barorreceptoras, de SHR machos e fêmeas.

Por fim, o estudo realizado por Tomassoni et al. ${ }^{1}$, certamente é um dos raros disponíveis, que relata dados morfológicos e propriedades de condução de nervo somato-motor e sensorial em SHR. Quatro técnicas, (microscopia de luz, imunoreatividade, imunohistoquímica e eletroneuromiografia) foram utilizadas para analisar o nervo isquiático de SHR e WKY, com 6 meses de vida. $\mathrm{O}$ estudo mostrou que o número total de fibras do nervo isquiático não sofre alteração, quando comparados ratos hipertensos e normotensos, mas a bainha de mielina se mostra significativamente reduzida nas fibras mais calibrosas (15-20 $\mu \mathrm{m})$, em SHR. Os autores concluem ainda, que as alterações morfológicas do nervo isquiático dos SHR provavelmente apresentam relevância funcional, uma vez que essas alterações são acompanhadas de diminuição da velocidade de condução. Um outro achado desse estudo foi a diminuição da porcentagem de grandes fibras mielínicas e um aumento da porcentagem das fibras 
A

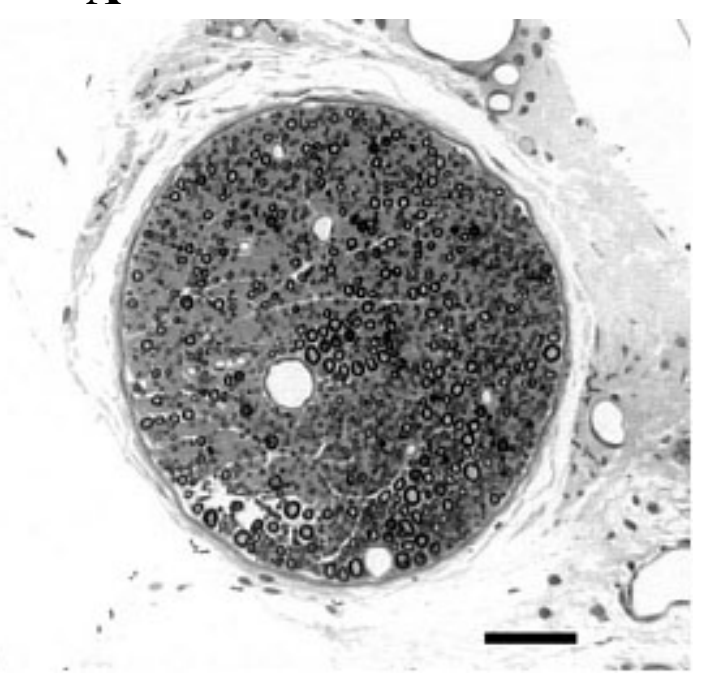

C

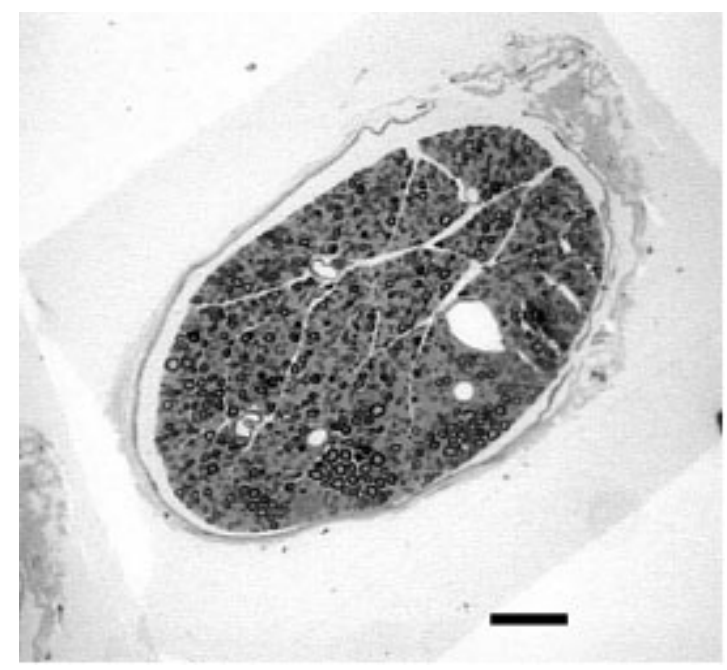

B

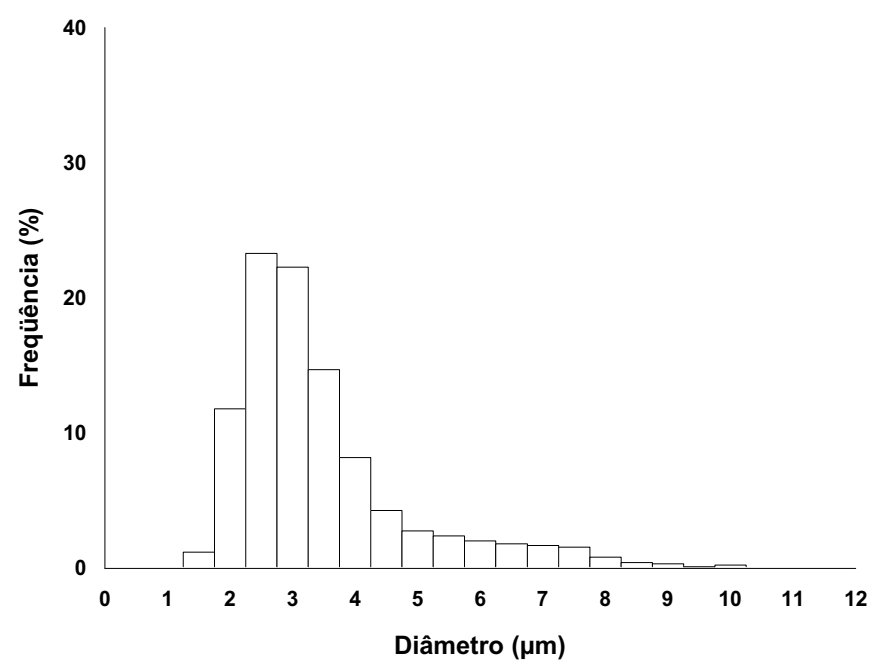

D

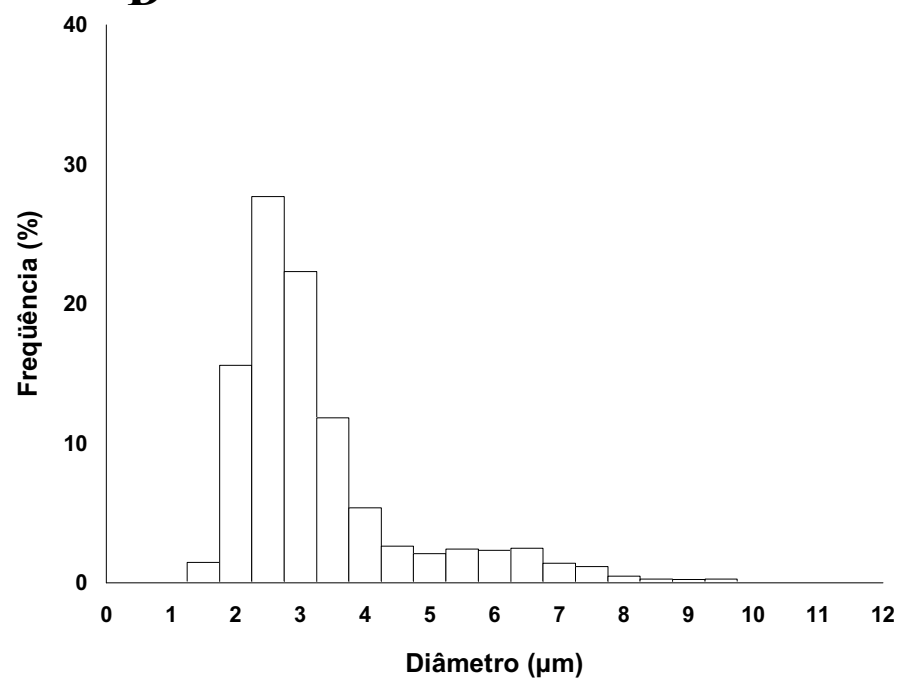

Figura 2: Secção transversal semi-fina (segmento proximal) de nervo vago de SHR macho (A), com a representação do histograma de distribuição dos diâmetros das fibras mielínicas (B). Notar que o nervo vago apresenta grande número de fibras mielínicas pequenas, sendo o histograma unimodal, com pico em 2,5 $\mu \mathrm{m}$. Não há diferença morfológica entre os nervos dos SHR machos $(A)$ e fêmeas (B). A distribuição dos histogramas de diâmetro das fibras mielínicas dos SHR fêmeas (D) também não difere da observada em machos. Barra de escala em A e C $=50 \mu \mathrm{m}$.

pequenas no nervo isquiático dos SHR. Esse achado corrobora nossas observações prévias ${ }^{42}$, que mostra uma tendência das fibras mielínicas do NDA de SHR apresentarem diâmetros médios menores e histograma deslocado para a esquerda, em comparação com os WKY. Os autores sugerem que, provavelmente, as modificações observadas no nervo isquiático dos SHR dependem do aumento da pressão arterial e não apenas de uma característica intrínseca dos ratos espontaneamente hipertensos.
Nessa linha, resultados preliminares do nosso laboratório, em secções transversais de nervos surais de SHR fêmeas (Figura 3), mostram que ele é arredondado ou oval, envolto por um perineuro bem definido, subdividido em fascículos, como descrito por Jerônimo et al ${ }^{48}$. No endoneuro, a sua citologia geral não difere daquela de outros nervos periféricos e, de modo geral, pode ser comparável com observações morfológicas realizadas em nervos sensitivos ${ }^{49 / 52} \mathrm{e}$ $\operatorname{mistos}^{49,53,54,55}$. 
Tabela III: Parâmetros morfométricos dos fascículos e das fibras mielínicas dos nervos vagos de SHR, lado direito, machos e fêmeas.

\begin{tabular}{|c|c|c|c|c|}
\hline & \multicolumn{2}{|c|}{ Machos $(\mathbf{N}=5)$} & \multicolumn{2}{|c|}{ Fêmeas $(\mathrm{N}=5)$} \\
\hline & Proximal & Distal & Proximal & Distal \\
\hline Área Fascicular $(\mu \mathrm{m} 2)$ & $77072 \pm 4389$ & $75301 \pm 3377$ & $65526 \pm 4679$ & $63124 \pm 1805^{*}$ \\
\hline Diâmetro Fascicular ( $\mu \mathrm{m})$ & $283 \pm 10$ & $265 \pm 26$ & $245 \pm 20$ & $268 \pm 6$ \\
\hline Número de fibras mielínicas & $1249 \pm 79$ & $1281 \pm 67$ & $1047 \pm 114$ & $1093 \pm 53$ \\
\hline Densidade de fibras mielínicas (fibras $/ \mathrm{mm} 2$ ) & $25303 \pm 1194$ & $24306 \pm 895$ & $26118 \pm 1882$ & $27808 \pm 908^{*}$ \\
\hline Diâmetro das fibras mielínicas ( $\mu \mathrm{m})$ & $3,27 \pm 0,08$ & $3,34 \pm 0,11$ & $3,21 \pm 0,06$ & $3,23 \pm 0,06$ \\
\hline Diâmetro dos axônios mielinizados ( $\mu \mathrm{m})$ & $1,53 \pm 0,07$ & $1,56 \pm 0,06$ & $1,56 \pm 0,03$ & $1,63 \pm 0,05$ \\
\hline Área da bainha de mielina $(\mu \mathrm{m} 2)$ & $9,57 \pm 0,36$ & $10,33 \pm 0,88$ & $8,97 \pm 0,48$ & $8,94 \pm 0,30$ \\
\hline Razão g & $0,48 \pm 0,02$ & $0,48 \pm 0,01$ & $0,50 \pm 0,01$ & $0,52 \pm 0,01$ \\
\hline
\end{tabular}
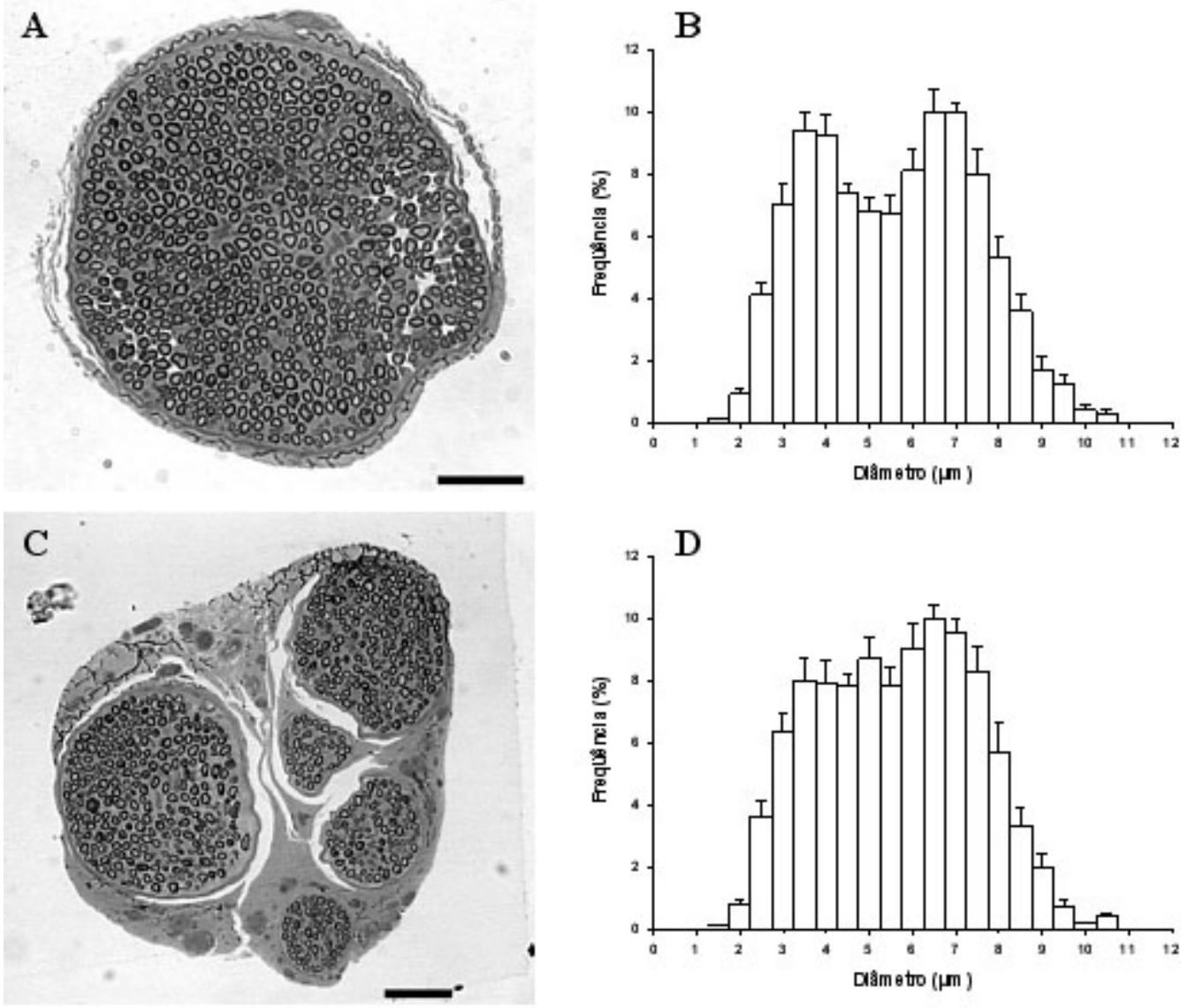

Figura 3: Secção transversal semi-fina de nervo sural, segmento proximal (A) de SHR fêmea, com a representação do histograma de distribuição dos diâmetros das fibras mielínicas (B). Notar que o nervo sural apresenta histograma de distribuição das fibras mielínicas bi-modal, com picos em 3,5 e 6,5 $\mu \mathrm{m}$. No segmento distal (C), notar a presença de vários fascículos, envolvidos por um epineuro evidente. A distribuição dos histogramas de diâmetro das fibras mielínicas no segmento distal (D) não difere da observada no segmento proximal. Barra de escala em A e C $=50 \mu \mathrm{m}$ 
A importância de se explorar morfometricamente diferentes segmentos do mesmo nervo está no fato de que existem descrições de alterações patológicas que são mais acentuadas distalmente ${ }^{48,56,57}$. O conhecimento do normal nos diversos segmentos do mesmo nervo, pode auxiliar na interpretação de achados patológicos desse nervo, principalmente em modelos experimentais de doenças, como é o caso do SHR para a hipertensão essencial. Com relação ao estudo de lateralidade, nosso dado é importante uma vez que, muitos estudos experimentais que utilizam lesões cirúrgicas do nervo isquiático e/ou seus ramos, utilizam os nervos da pata contra-lateral como controle ${ }^{58,59.60}$. Embora pareça lógica essa utilização, cuidados na interpretação dos resultados obtidos devem ser tomados pois existem descrições na literatura de assimetria lateral entre nervos periféricos ${ }^{612,62,63}$.

Esse é o primeiro relato sobre os parâmetros morfométricos de nervos surais e suas fibras mielínicas em SHR (Tabela IV). Dentre os componentes celulares do espaço endoneural dos nervos surais de SHR, que não as fibras nervosas, as células de Schwann puderam ser facilmente distinguidas pelas suas relações com os axônios. É de amplo conhecimento que os componentes do espaço endoneural apresentam papel fundamental nos processos de regeneração e reparo que seguem uma lesão nervosa. Contagens do número de núcleos do espaço endoneural de nervos periféricos, tanto humanos ${ }^{64}$ quanto em animais de laboratório ${ }^{64,65,66}$ evidenciaram um predomínio de núcleos de células de Schwann em relação a outros tipos de núcleos celulares, em proporções que variaram de $45 \%$ até $95 \%$. Nos nervos surais desse estudo, outros núcleos, exceto os de células de Schwann, foram encontrados com pouca freqüência. O número de núcleos de células de Schwann foi de aproximadamente 15, dispersos em uma densidade de 349 núcleos $/ \mathrm{mm}^{2}$ de área fascicular, sem diferenças entre segmentos e lados.

\section{AGRADECIMENTOS}

Os autores agradecem o excelente auxílio técnico de Maria Teresa Picinato Maglia, Maria Cristina Lopes Schiavoni, Aracy Edwirges Vieira da Silva Dias, Antônio Renato Meirelles e Silva e João Carlos Araújo Ribas.

Apoio financeiro FAPESP, processos 02/09406-5, 04/01390-8, 04/09139-2, CNPq, processo 501230/2003-3 e FAEPA, processos 68/2003 e 393/2005.

\begin{tabular}{|c|c|c|c|c|}
\hline & \multicolumn{2}{|c|}{ Direito $(\mathrm{N}=5)$} & \multicolumn{2}{|c|}{ Esquerdo $(\mathrm{N}=5)$} \\
\hline & Proximal & Distal & Proximal & Distal \\
\hline Área Fascicular $\left(\mu \mathrm{m}^{2}\right)$ & $50056 \pm 3725$ & $34881 \pm 6726$ & $48873 \pm 3523$ & $56803 \pm 5415$ \\
\hline Diâmetro Fascicular $(\mu \mathrm{m})$ & $256 \pm 17$ & $249 \pm 33$ & $295 \pm 35$ & $398 \pm 37$ \\
\hline Número de fibras mielínicas & $861 \pm 14$ & $589 \pm 73$ & $855 \pm 81$ & $792 \pm 53$ \\
\hline Densidade de fibras mielínicas (fibras $/ \mathrm{mm}^{2}$ ) & $17595 \pm 1324$ & $17972 \pm 1511$ & $17431 \pm 835$ & $14286 \pm 1172$ \\
\hline Diâmetro das fibras mielínicas ( $\mu \mathrm{m})$ & $5,17 \pm 0,19$ & $5,16 \pm 0,14$ & $5,26 \pm 0,14$ & $5,43 \pm 0,14$ \\
\hline Diâmetro dos axônios mielinizados $(\mu \mathrm{m})$ & $2,49 \pm 0,18$ & $2,49 \pm 0,16$ & $2,53 \pm 0,12$ & $2,71 \pm 0,10$ \\
\hline Área da bainha de mielina $\left(\mu \mathrm{m}^{2}\right)$ & $21,46 \pm 1,32$ & $20,34 \pm 1,02$ & $21,37 \pm 0,96$ & $22,92 \pm 1,06$ \\
\hline Razão $g$ & $0,47 \pm 0,02$ & $0,48 \pm 0,02$ & $0,47 \pm 0,01$ & $0,48 \pm 0,01$ \\
\hline
\end{tabular}


Fazan VPS, Kalil ALR, Alcântara ACL, Genari AB, Tavares MR, Rodrigues AR, Salgado HC. Spontaneously hypertensive rats and peripheral neuropathies. Medicina (Ribeirão Preto) 2006; 39 (1): 39-50.

Abstract: Type of the study: The present study is a literature review about the spontaneous hypertension animal model, and the consequences of the hypertension to the peripheral nervous system, somatic and autonomic.

Importance of the topic: Hypertension is the main risk factor to stroke and vascular dementia, due to important cerebrovascular changes that may lead to cerebral microaneurysms, infarction and acute ischemia. The main central nervous system changes due to hypertension are the reduction of the cerebral volume, increase of the ventricles volume and loss of neurons. Moreover, hypertension causes renal alterations and other pathologies that might sustain the high blood pressure, the tachycardia and the elevation of the peripheral vascular resistance. The spontaneously hypertensive rat (SHR) is recognized as an excellent model of the human essential hypertension. Nevertheless, despite that this animal model has been widely explored in terms of physiological studies, morphological studies, when available, are limited to the vessels. Even when peripheral nerves are being explored, the epineural, perineural and endoneural vessels are the subject of the studies. Information on the alterations of the myelinated and unmyelinated fibers in this model of hypertension is scanty.

Comments: Recently, we studied the morphology and morphometry of the aortic depressor nerve (ADN) in SHR and our results have demonstrated a reduction of the myelinated fibers size and a reduction of the number and size of the unmyelinated fibers, compared to the normotensive controls Wistar-Kyoto. Another recent study from our laboratory showed that, despite the significantly higher blood pressure and heart rate on male SHR, compared to female SHR, there are no morphological differences on the vagus nerves between males and females. Also, we have described the morphological and morphometric characteristics of the sural nerve in SHR, thus providing morphological background for further functional studies.

Keywords: Hypertension, Experimental. Hypertension Neuropathies. Neuropathies, Autonomic. Aortic Depressor Nerve. Sural Nerve. Vagus Nerve.

\section{REFERÊNCIAS}

1 - Tomassoni D, Traini E, Vitaioli L, Amenta F. Morphological and conduction changes in the sciatic nerve of spontaneously hypertensive rats. Neurosci Lett 2004; 362: 131-5.

2 - Zicha J, Kunes J. Ontogenetic aspects of hypertension development: analysis in the rat. Physiol Rev 1999; 79: 1227-82.

3 - Dickhout JG, Lee RMKW. Structural functional analysis of small arteries from young spontaneously hypertensive rats. Hypertension 1997; 29: 781-9.

4 - Okamoto K, Aoki K. Development of a strain of spontaneously hypertensive rats. Jpn Circ J 1963; 27: 282-93.

5 - Okamoto K, Tabei R, Fukushima M, Nosaka S, Yamori Y, Ichijima K, Haebara H, Matsumoto M, Maruyama T, Suzuki Y, Tamegai M. Further observations of the development of a strain of spontaneously hypertensive rats. Jpn Circ J 1966; 30: 703-16.

6 - Undenfreind S, Spector S. Spontaneously hypertensive rat. Science 1972; 176: 1155-6.

7 - Trippodo NC, Frohlich ED. Similarities of genetic (spontaneous) hypertension: man and rat. Circ Res 1981; 48: 309-19.
8 - Mullins MM, Banks RO. Age-related changes in $\mathrm{Na}+$ excretion in saline-loaded spontaneously hypertensive rats. Am J Physiol 1976; 231: 1364-70.

9 - Sinaiko AR, Mirkin BL. Isoproterenol-evoked renin release from the in situ perfused kidney. Dose-response characteristics in spontaneously hypertensive and normotensive Wistar rats. Circ Res 1978; 42: 381-5.

10 - Dickhout JG, Lee RMKW. Blood pressure and heart rate development in young spontaneously hypertensive rats. Am J Physiol Heart Circ Physiol 1998; 274: 794-800.

11 - Louis WJ, Tabei R, Spector S. Effects of sodium intake on inherited hypertension in the rat. Lancet 1971; 2: 1283-6.

12 - Yamori Y, Matsumoto M, Yamabe H, Okamoto K. Augmentation of spontaneou hypertension by chronic stress in rats. Jpn Circ J 1969; 33: 399-409.

13 - Hallback M, Isaksson O, Noresson E. Consequences of myocardial structural adaptation on left ventricular compliance and the Frank-Starling relationship in spontaneously hypertensive rats. Acta Physiol Scand 1975; 94: 259-70.

14 - Lais LT, Shaffer RA, Brody MJ. Neurogenic and humoral factors controlling vascular resistance in the spontaneously hypertensive rat. Circ Res 1974; 35: 764-74. 
15- Potts JT, Mckeown KP Shoukas AA. Reduction in arterial compliance alters carotid baroreflex control of cardiac output in a model of hypertension. Am J Physiol 1998; 274: H1121-31.

16- Pfeffer MA, Frohlich ED. Hemodynamics of spontaneously hypertensive rats. I. Effects of pressure elevation. Am J Physiol 1973; 224: 1066-71.

17- Frohlich ED. Essential hypertension. Pathophysiological mechanisms and therapy. Arch Intern Med 1977; 137: 772-5.

18- Head GA. Cardiac baroreflexes and hypertension. Clin Exp Pharmacol Physiol 1994; 21: 791-802.

19- Matsuoka H, Nakata M, Kohno K, Koga Y, Nomura G, Toshima $\mathrm{H}$, Imaizumi T. Chronic L-arginine administration attenuates cardiac hypertrophy in spontaneously hypertensive rats. Hypertension 1996; 27:14-8, 1996.

20- Thomas CJ, Rankin AJ, Head GA, Woods RL. ANP enhances bradycardic reflexes in normotensive but not spontaneously hypertensive rats. Hypertension 29:1126-32, 1997.

21- Judy WV, Watanabe AM, Henry DP, Besch HR Jr, Murphy WR, Hockel GM. Sympathetic nerve activity: role in regulation of blood pressure in the spontaenously hypertensive rat. Circ Res 1976; 38: 21-9.

22- Lundin S, Ricksten SE, Thoren P. Interaction between "mental stress" and baroreceptor reflexes concerning effects on heart rate, mean arterial pressure and renal sympathetic activity in conscious spontaneously hypertensive rats. Acta Physiol Scand 1984; 120:273-81.

23- Folkow B. Early structural changes in hypertension: pathophysiology and clinical consequences. J Cardiovasc Pharmacol 1993; 22 (Suppl 1): 1-6.

24- Overbeck HW, Johnston RF. Experimental variation in vascular response studies. Proc Soc Exp Biol Med 1972; 141:1041-3.

25- Prewitt RL, Chen II, Dowell R. Development of microvascular rarefaction in the spontaneously hypertensive rat. Am J Physiol 1982; 243: H243-51.

26- Greenberg S, Bohr Df. Venous smooth muscle in hypertension. Enhanced contractility of portal veins from spontaneously hypertensive rats. Circ Res 1975; 36(6 Suppl 1): 208-15.

27- Frohlich ED, Pfeffer MA .Adrenergic mechanisms in human hypertension and in spontaneously hypertensive rats. Clin Sci Mol Med 1975; 2: 225s-38s.

28- Van Gorp AW, Schenau DS, Hoeks AP, Boudier HA, De Mey JG, Reneman RS. In spontaneously hypertensive rats alterations in aortic wall properties precede development of hypertension. Am J Physiol Heart Circ Physiol 2000, 278:H1241-7.

29- Judy WV, Farrell SK. Arterial baroreceptor reflex control of sympathetic nerve activity in the spontaneously hypertensive rat. Hypertension 1979; 1: 605-14.
30- Morrison SF, Whitehorn D. Baroreceptor reflex gain is not diminished in spontaneous hypertension. Am J Physiol 1982; 243: R500-5.

31- Touw KB, Haywood JR, Shaeffer RA, Brody MJ. Contribution of the sympathetic nervous system to vascular resistance in conscious young and adult spontaneously hypertensive rats. Hypertension 1980; 2: 408-18.

32- Sen S, Tarazi RC, Khairallah PA, Bumpus FM. Cardiac hypertrophy in spontaneously hypertensive rats. Circ Res 1974; 35: $775-81$.

33- Sen S, Tarazi RC, Bumpus FM. Effect of converting enzyme inhibitor (SQ14,225) on myocardial hypertrophy in spontaneously hypertensive rats. Hypertension 1980; 2: 169-76.

34- Pfeffer MA, Pfeffer JM, Frohlic ED. Pumping ability of the hypertrophying left ventricle of the spontaneously hypertensive rat. Circ Res 1976; 38: 423-9.

35- Weiss L, Lundgren Y. Left ventricular hypertrophy and its reversibility in young spontaneously hypertensive rats. Cardiovasc Res 1978; 12: 635-8.

36- Kramer JM, Waldrop TG. Spontaneously hypertensive rats exhibit altered cardiovascular and neuronal responses to muscle contraction. Exp Physiol 2001; 86: 717-24.

37- Sabbatini M, Vega JA, Amenta F. Peripheral nerve vascular changes in spontaneously hypertensive rats. Neurosci Lett 1996; 217: 85-8.

38- Persson K, Pandita RK, Spitsbergen JM, Steers WD, Tuttle JB, Andersson KE. Spinal and peripheral mechanisms contributing to hyperactive voiding in spontaneously hypertensive rats. Am J Physiol Regul Integr Comp Physiol 1998; 275: R1366-73.

39- Zanchetti A, Mancia G. Structural cardiovascular adaptation and the consequence for baroreflexes. Hypertension 1984; 6: $93-9$

40- Widdop RE, Verberne AJ, Louis WJ, Jarrott B. The effect of ketanserin on cardiovascular reflexes in conscious normotensive and spontaneously hypertensive rats. Eur J Pharmacol 1990; 186: 17-28.

41- Head GA, Adams MA. Time course of changes in baroreceptor reflex control of heart rate in conscious SHR and WKY: contribution of the cardiac vagus and sympathetic nerves. Clin Exp Pharmacol Physiol 1988; 15: 289-92.

42- Fazan VPS, Fazan Jr. R, Salgado HC, Barreira AA. Aortic depressor nerve myelinated fibers morphology in normotensive Wistar-Kyoto and spontaneously hypertensive rats. J Auton Nerv Sys 1999; 77: 133-9.

43- Fazan VPS, Salgado HC, Barreira AA. Aortic depressor nerve unmyelinated fibers in spontaneously hypertensive rats. Am J Physiol Heart Circ Physiol 2001; 280: H1560-4.

44- Takaeda K, Hayashi J, Itoh H, Hirata M, Nakata T, Oguro M, Kawasaki S, Sasaki S, Nakagawa M. Transection of aortic nerve fails to raise blood pressure in spontaneously hypertensive rats. Cardiovasc Res 1989; 23: 573-6. 
45- Thorém P, Saum WR, Brown AM. Characteristics of rat aortic baroreceptors with nonmedullated afferent nerve fibers. Circ Res 1977; 40: 231-7.

46- Fazan VPS, Salgado HC, Reis GC, Barreira AA. Relation between myelin area and axon diameter in the aortic depressor nerve of spontaneously hypertensive rats. J Neurosci Meth 2005; 148: 130-6.

47- Minwegen P, Friede RL. Conduction velocity varies with osmotically induced changes of the area of the axons' profile. Brain Res 1984; 297: 105-13.

48- Jeronimo A, Jeronimo CAD, Rodrigues Filho OA, Sanada LS, Fazan VPS. Microscopy anatomy of the sural nerve in the postnatal developing rat: a longitudinal and lateral symmetry study. J Anat 2005; 206: 93-9.

49- Causey G, Barton AA. The cellular content of the endoneurium of peripheral nerve. Brain 1959; 82: 594-8.

50- Ferriere G, Denef JF, Rodrigues J, Guzzetta F. Morphometric studies of normal nerves in children. Muscle Nerve 1985; 8: 697-704.

51- Jacobs JM, Love S. Qualitative and quantitative morphology of humam sural nerve at different ages. Brain 1985; 108: 897-924.

52- Fazan VPS, Salgado HC, Barreira AA. A descriptive and quantitative light and electron microscopy study of the aortic depressor nerve in normotensive rats. Hypertension 1997; 30: $693-8$

53- Friede RL, Samorajski T. Relation between the number of myelin lamellae and axon circumference in fibers of vagus and sciatic nerves of mice. J Comp Neurol 1967; 130: 223-32.

54- Wozniak W, O'Rahilly R, Bruska M. Myelination of the human fetal phrenic nerve. Acta Anat 1982;112: 281-96.

55- Fazan VPS, Ma X, Chapleau MW, Barreira AA. Qualitative and quantitative morphology of renal nerves in C57BL/6J mice. Anat Rec 2002; 268: 399-404.
56- Grover-Johnson N, Spencer PS. Peripheral nerve abnormalities in aging rats. J Neuropathol Exp Neurol 1981; 40: 155-65.

57- Thomas PK, King RHM, Sharma AK. Changes with age in the peripheral nerves of the rat. Acta Neuropathol 1980; 52: 1-6.

58- Kim DH, Connolly SE, Kline DG, Voorhies RM, Smith A, Powell M, Yores T, Daniloff JK. Labeled Schwann cell transplants versus sural nerve grafts in nerve repair. J Neurosurg 1994; 80: $254-60$.

59- Nagamatsu M, Sugimura K, Aoki S, Takahashi A. Morphometric analysis of the sural nerve in experimental dorsal rhizotomy in the rats. J Neurol Sci 1993; 116: 170-5.

60- Kovacic U, Sketelj J, Bajrovic FF. Sex-related difference in collateral sprouting of nociceptive axons after peripheral nerve injury in the rat. Exp Neurol 2003; 184: 479-88.

61- Fraher JP. Myelin-axon relationships in the rat phrenic nerve, longitudinal variation and lateral asymmetry. J Comp Neurol 1992; 323: 551-7.

62- Song A, Tracey DJ, Ashwell KW. Development of the rat phrenic nerve and the terminal distribution of phrenic afferents in the cervical cord. Anat Embryol 1999; 200: 625-43.

63- Rodrigues Filho OA, Fazan VPS. Streptozotocin induced diabetes as a model of phrenic nerve neuropathy in rats. J Neurosci Meth 2006; 151: 131-8.

64- Gamble HJ, Eames R. An electron microscope study of the connective tissues of human peripheral nerve. J Anat 1964; 98: $655-63$.

65- Causey G, Barton AA. The cellular content of the endoneurium of peripheral nerve. Brain 1959; 82: 594-8.

66- Thomas PK. The connective tissue of peripheral nerve: an electron microscope study. J Anat 1963; 97: 35-44. 